\title{
Research on the Dynamic Model of 2.5D Fluid Structure Interaction of Gravity Dam and Simulation of Seismic Stress Response
}

\author{
LI Yiran ${ }^{1,2}$, XU Menghua ${ }^{2, ~}{ }^{*}$, SUN Hao ${ }^{2}$, Xiao Genfeng ${ }^{2}$ \\ 1.Hunan Province Key Laboratory of Key Technologies for Water Power Resources \\ Development,Hunan,Changsha,410000; \\ 2.Institute of Civil engineering and Transportation ,South China University of \\ Technology,Guangdong,Guanghzou,510640; \\ *corresponding author's e-mail: mhxu@scut.edu.cn.
}

Keyword: Dynamic Model, critical engineering, economic development

Abstract: The seismic safety of the dam involves the safety of people's life and property, and even affects the overall safety of the national economy. One of the key of technical issues in this subject is the more complete fluid-structure interaction(fsi) theory and the more accurate calculation method. In order to solve the problem of dynamic fluid-structure interaction in gravity dam, a $2.5 \mathrm{~d}$ semi-dynamic theoretical model and a numerical solution method are established by using fluid slice method. The numerical experiment of seismic sress response is compared with threedimensional model. The results show that the stress response of the $2.5 \mathrm{~d}$ semi-dynamic model of gravity dam can well reflect the fluid- structure interaction behavior and effect of seismic gravity of concrete gravity dam.

\section{Introduction}

Most areas of China belong to the frequently-occurring intense earthquake area, and under seismic action, the aseismic safety of many engineering systems involving FSI such as water retaining structures like gate dam, water transport structures like aqueduct[1] and pipe work, liquid storage tank and so on, gains wide attention from workers of earthquake engineering[2]. When this kind of engineering structures suffer an intense earthquake, it will cause not only direct engineering loss, but also secondary disasters what are hard to expect and threat to people's lives and properties, even a great impact on national economic development. Since 1960s and 1970s, Pacoima[3] arch dam in America, Koyna gravity dam in India, and Sefid-Rud Dam in Brazil all with a height over a hundred meters suffered an earthquake $(\mathrm{ms} \geq 6)$, which are typical living examples of dam damage. And now in China, the high concrete dams what are being built or have been built are most situated in the southwest where earthquakes happen intensely. Therefore, aseismic safety evaluation about dam has always been a research project attracting much attention. Especially after Wenchuan earthquake [4] in 2008, the aseismic safety of dam raises a high attention of broad scholars and society again. So higher requirement for critical engineering and lifeline engineering is necessary.

The key to this project is to have a fairly complete FSI theory and a calculation method with a good accuracy. And interaction of dam- reservoir system when earthquake is a typical FSI project. Since H.M.Westergaard published his famous paper Water pressure on dams during earthquakes[5], because of the significance of this project in engineering practice, it has caught many researches' attention for a long time. On retrospect, the research course can be divided into two stages: before the 1960s, the research mainly focused on analytic solution while the solution only possessed theoretical significance on account of calculation being simplified much; after the 1960s, the development of computer technology and promoted application of FEM have provided powerful research tools to solve dam-reservoir coupling problem, which allows to adopt more realistic calculation model considering complicated working condition.

Analytical method[6], although asking to simplify problem as much as possible, is applicable to theoretic exploration because it has less calculation amount. For this reason, there are still some scholars[7] devoting themselves to this aspect. However, if the computed region is rather complex, 
it is very hard to find a trial function satisfying the boundary conditions, which will greatly decrease the efficiency and accuracy of calculation.

FEM applied to solving FSI problem is presented first by The finite element method[8], a noted book by iZeknieMcz. After developing from low level to high level and simplicity to complexity, FEM is extensively and profoundly practical in engineering projects, as the most efficient analytic method. According to FSI research project, large finite element software is usually used to build theoretical models and do simulation calculation and analysis, where the adopted models are most 2D and 3D models. Ghaedi[9] builds 2D model to analyze the concrete dam under seismic action. And Puigdellivol[10] also builds a 2D model to study a topology optimization method for laminated busbars in power converters and he points out that Comparison with 3D FEM proves the 2-D approach to be faster while remaining accurate and a perfect method. However, it is obvious that considering the complexity of large engineering projects like dam, there are too many factors what should be taken into account that 2D theoretical model cannot meet the accuracy requirement. In Crosta' study about collapse and rockslide-reservoir interaction, a fully 3D rockslide-water reservoir simulation has been considered to provide a more realistic simulation. But 3D simulation calculation of large engineering has a high requirement for computer and that will takes a long time, which greatly decrease the calculation efficiency.

However, 2.5D simulation calculation can increase the accuracy and efficiency of calculation at the same time. This 2.5D theory has extensive application and research in fields of shipping engineering, hydraulic engineering, biological blood vessels and so on. And some research results have been achieved in those areas that have good simulation effect. But at present, the research of $2.5 \mathrm{D}$ theory in hydraulic engineering is mainly about large aqueduct, little attention is paid to gravity dam. However finding a rational and effective FSI dynamic calculation methods is of great significance for the large-scale seismic dynamic analysis of gravity dam.

Therefore, the main objectives of this study are (1) to present 2.5D fluid-structure interaction dynamic model of gravity dam under seismic action, (2) to compare the calculation results in numerical test method with that by 3D model, (3) to verify the feasibility, rationality and accuracy of .25D simulation, (4) to simply discuss how to build a $2.5 \mathrm{D}$ model with the best simulation.

\section{Model control equation}

The FSI 2.5D theory means that the model is consist of 3D solid structure and 2D fluid structure, where 2D fluid structure simulates the FSI effect between structure and fluid.

\section{Governing equation of structure}

Dynamic governing equation of dam body

$$
[M]\left\{[ C ] \left\{l \&+[K]\{u\}=\left\{F_{s}(t)\right\}\right.\right.
$$

In the above equation, $\&$ mean acceleration, velocity and displacement column vector in system; $F_{s}(t)$ means instantaneous load vector on system, including water pressure acted on 2D dam body by fluid, which is determined by equation of fluid motion; $K$ means structure stiffness matrix; $C$ means structure damping matrix.

\section{Governing equation of fluid domain}

The ALE (Arbitrary Lagrange Euler) description is introduced here. The ALE Kinematic description is perfectly practical in solving the large-scale amplitude sloshing problem of fluid on free surface. N-S equation and continuity equation under the ALE description are shown as follows:

$$
\left\{\begin{array}{l}
\rho \rho a \cdot \nabla \iota \&-\nabla \tau-f=0 \\
\nabla \cdot \imath=0
\end{array}\right.
$$


In the equation, $\rho$ means mass density; $u$ means displacement; $\iota \mathcal{L}_{\text {and }}$ mean the first and

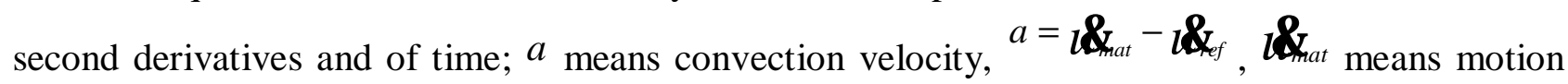
velocity of material; $1 \mathbb{S}$ means motion velocity of eulerian grid; $f$ means external force, for the nodes on coupling interface, includes coupling force; $\tau$ means stress tensor of fluid.

\section{Fluid free surface}

Water free surface is nonpenetrating boundary here, which necessitates meeting the requirement of motion boundary condition and dynamic boundary condition as follows:

$$
\left\{\begin{array}{l}
\partial S / \partial t+v_{i} S_{, i}=0 \\
\tau_{i j} n_{j}=\left[-p_{0}+\sigma\left(1 / R_{1}+1 / R_{2}\right)\right] n
\end{array}\right.
$$

In this equation, $V_{i}$ means velocity vector of fluid surface, ${ }^{p_{0}}$ means external pressure exerted on free surface, $\sigma$ means surface tension, ${ }^{n}$ means unit normal vector of free surface, ${ }^{R}$ and $R_{2}$ means curvature radiuses on fluid free curved surface in the two horizontal coordinate directions. The large-scale amplitude sloshing non-linear characteristics of free surface are taken into amount of solution.

\section{Coupling condition}

Water couples on interaction surface with dam body, and velocity and interaction are continuous on coupling boundary surface, which meets the requirement of continuous condition as follows:

$$
\left\{\begin{array}{l}
\iota \mathcal{\&}=\imath \mathbb{\&} \\
s_{f} \cdot n_{f}=s_{s} \cdot n_{s}
\end{array}\right.
$$

In this equation, $s_{f}$ and $s_{s}$ mean stress of water and dam body structure on coupling surface; ${ }_{f}$ and $n_{s}$ mean normal vectors of water and dam body structure on coupling surface with direction outward.

\section{Case study}

\section{Introduction of studied dam}

The concrete dam is $120 \mathrm{~m}$ high with upstream slope vertical, downstream slope gradient 0.75 , crest dam 1.5 times the length of dam height and 0.1 times the breadth of dam height. Dam body of gravity dam uses homogeneous concrete material, where elastic modulus of concrete $\mathrm{E}$ equals $2.85 \times 1010 \mathrm{~Pa}$, Poisson's ratio v equals 0.15 , density d equals $2600 \mathrm{Kg} / \mathrm{m} 3$, tensile strength $\mathrm{ft}$ equals $1.96 \times 106 \mathrm{~Pa}$ and compressive strength fc equals $2.2 \times 107 \mathrm{~Pa}$.Fluid uses water adopting laminar flow model whose parameters are shown as follows: density equals $1000 \mathrm{Kg} / \mathrm{m} 3$, viscous coefficient equals 0.002 ( $\mathrm{Pa} \cdot \mathrm{s}$ ), bulk elastic modulus equals $1000 \mathrm{MPa}$,and the fluid is set as incompressible liquid with the height of $108 \mathrm{~m}$ and the length of $240 \mathrm{~m}$. The seismic wave adopts Koyna earthquake wave of 10s, and the wave is shown in Fig.1. 


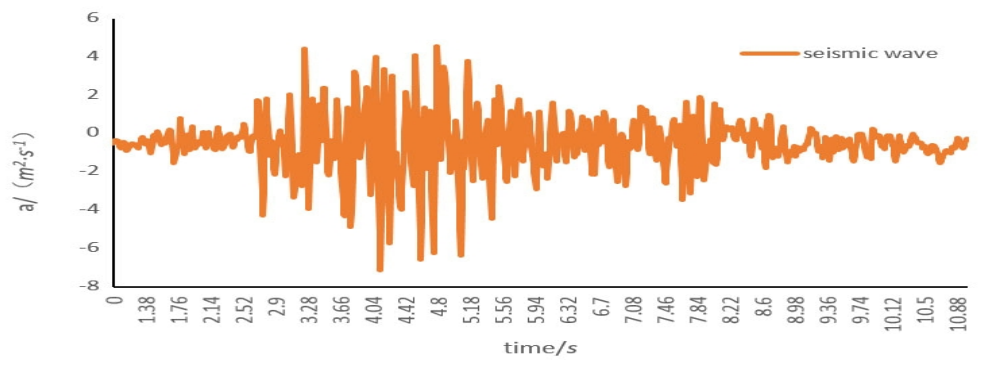

Fig.1 Koyna earthquake wave

\section{Model design}

In this model, dam body was 3D structure model and was divided into several 3D solid substructures according to the characteristics of structure and load, the FSI effect was transferred by setting up corresponding 2D dam shells between dam body sections of adjacent substructures; fluid was 2D fluid model, whose 2D shells were equally distributed on the dam surface with the same amounts of shells as 2D dam shells and corresponding locations with 2D dam shells.

Due to concrete gravity dam, the adopted numerical solution characteristics of FSI 2.5D theoretical model was as follows:

(1) The 3D solid elements were applied to dam body structure which were set gravity and Rayleigh damping up and exerted seismic load in dynamic response analysis;

(2) The 2D solid elements were applied to fluid whose amount and location of 2D shells corresponded with 2D dam shells, and the model was built with top surface as free surface, surface contacting dam as FSI surface, surface on the inverse side as uniform surface and bottom surface as wall surface;

(3) At present the FSI calculation between 3D model and 2D model cannot happen in finite element calculation grogram, which necessitates inserting 2D dam shells into 3D dam to fluidstructure interact with 2D fluid structure, making the material of 2D dam shells linear elastic material with no rigidity to avoid impacting the rigidity of dam body and at the same time contribute to transfer the action of fluid for dam body, setting constraint at the bottom of $2 \mathrm{D}$ dam shells and dam body and on the two side dam surfaces to restrain the displacement of dam bottom and the deformation of side surfaces .

The calculation results of 2.5D models with $6,12,24$ shells were all compared with that by 3D model. And the 2.5D model with 6 shells is shown in Fig.2 (2.5D models with 12 and 24 shells only had more equally spaced shells):

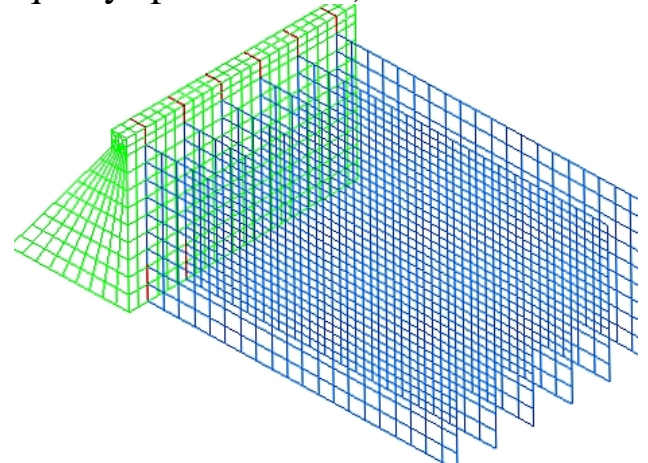

Fig.2 2.5D model with 6 shells

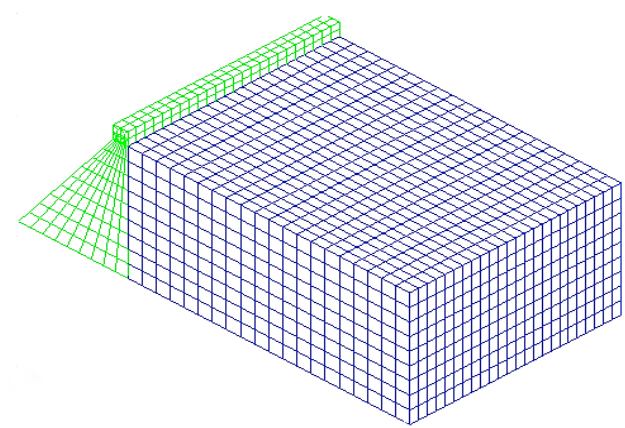

Fig. 3 3D model

The 3D model had the same parameter set as that of 2.5D models, which is shown in Fig.3: 


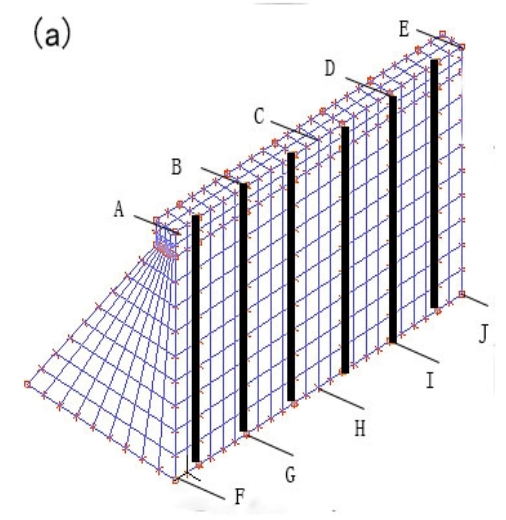

(b)

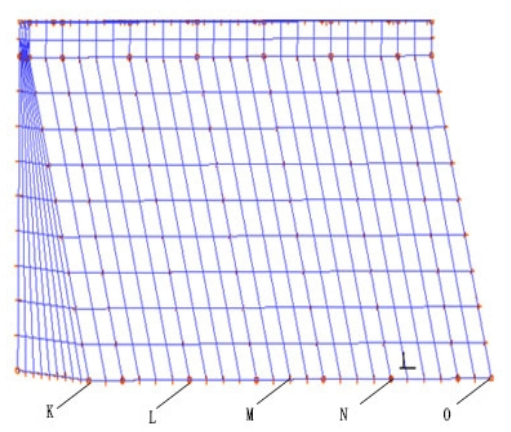

Fig.4 Picked points

The test picked up the stress data of points K, L, M, N, O on downstream sideline of dam bottom to compare. The positions of points K,L,M,N and $\mathrm{O}$ are shown in Fig.4.

\section{Result and discussion}

As shown in the figure, fig. 5 compares the sress of $\mathrm{M}$ and $\mathrm{L}$ points. Tab. 1 shows the comparison of the maximum absolute error of the first three stress peaks. (6-shellls, 12-shells,24-shells mean the 2.5D models with 6,12,24 shells), Tab.2 shows the comparison of mean absolute relative errors of stress.

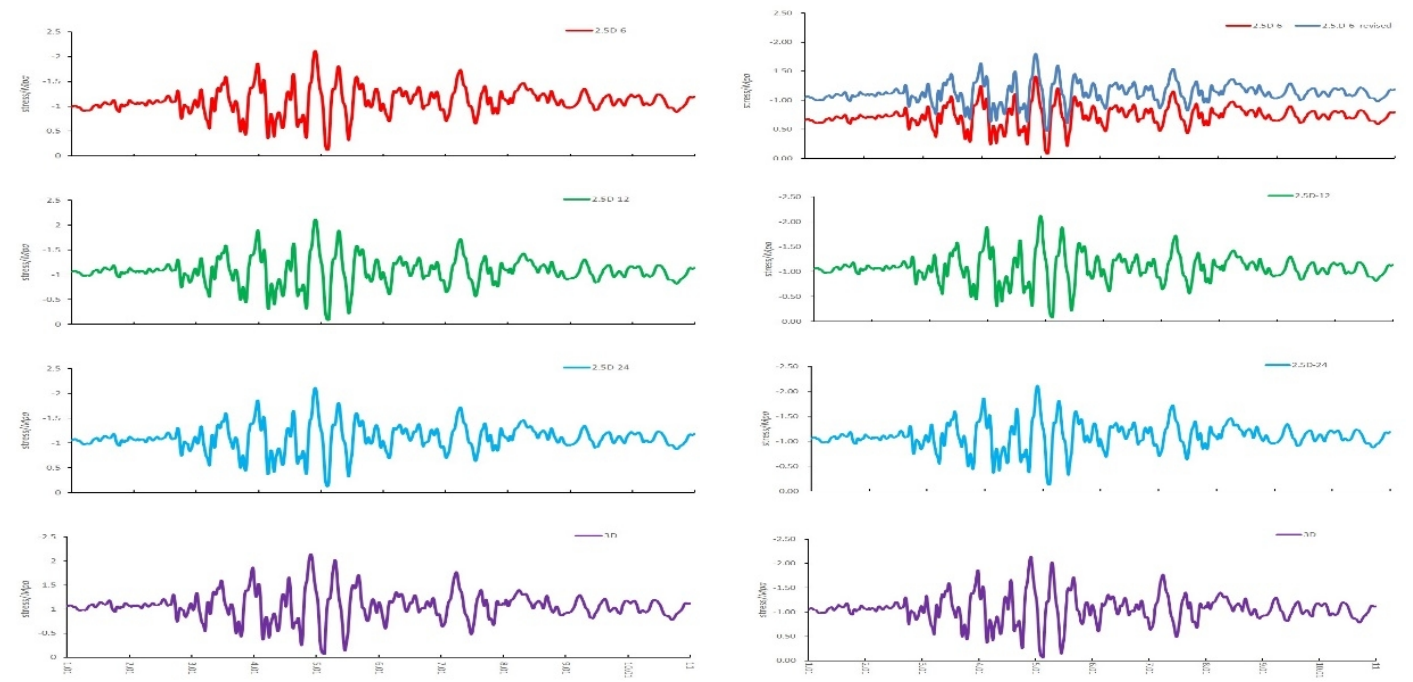

Fig.5 Comparison of stress of point $\mathrm{M}$ and point $\mathrm{L}$

Tab.1 Comparison of absolute relative errors of the first three stress peaks

Point B of 6 Point B of 12 Point B of 24 Point C of $6 \quad$ Point C of 12 Point C of 24

\begin{tabular}{lllllll}
\hline first peak & $34.04 \%$ & $1.06 \%$ & $1.18 \%$ & $1.03 \%$ & $1.16 \%$ & $1.29 \%$ \\
second peak & $34.19 \%$ & $0.53 \%$ & $1.26 \%$ & $1.23 \%$ & $0.61 \%$ & $1.33 \%$ \\
third & $34.04 \%$ & $1.90 \%$ & $1.34 \%$ & $1.03 \%$ & $2.00 \%$ & $1.44 \%$ \\
peak & & & &
\end{tabular}

Tab.2 Comparison of mean absolute relative errors of stress

\begin{tabular}{llllll}
\hline & Point K & Point L & Point M & Point N & Point L \\
\hline 6-shells & $12.28 \%$ & $62.39 \%$ & $12.28 \%$ & $62.26 \%$ & $12.76 \%$ \\
12-shells & $6.59 \%$ & $6.54 \%$ & $6.59 \%$ & $6.56 \%$ & $6.62 \%$ \\
24-shells & $10.80 \%$ & $10.79 \%$ & $10.80 \%$ & $10.90 \%$ & $10.99 \%$ \\
\hline
\end{tabular}


As shown in fig. 5, shows the stress response tendency of all $2.5 \mathrm{~d}$ model and the $3 \mathrm{~d}$ model is roughly same of point $\mathrm{M}$, However, the stress responses of 6-shells model at $\mathrm{L}$ point deviate significantly from the 3D model, while those of other models agree well with the 3D results.

Considering the static calculation error influence on the result of the dynamic stress response, the dynamic stress response is corrected by compensating the static calculation error in the dynamic stress response.It can be seen from fig. 5 that the stress response of the point $\mathrm{L}$ of the 6 -shells $2.5 \mathrm{~d}$ model after modification is similar to that of the $3 \mathrm{~d}$ model. Therefore, it is still possible to obtain the accuracy and reliability of the dynamic calculation analysis of $2.5 \mathrm{~d}$ model. The dynamic calculation results of the stress are still roughly the same as that of the $3 \mathrm{~d}$ model.

From Tab.1, it can be seen that the stress response errors of models with 6 shells at point $\mathrm{L}$ are abnormally large (still caused by the static calculation error), and the difference between the remaining errors is not large. The average error about the stress response of 12 shells models from Tab.2 is the smallest. Because the mean absolute errors of $2.5 \mathrm{~d}$ model with 12 -shells is smallest and its data process is similar to that of 3D model and the average error is the smallest, it appeared that the model with 12 shell had the comparatively better simulation.

\section{Conclusion}

This paper presented 2.5D model of concrete gravity dam to compare it with 3D model in test result to verify the correctness and rationality of solving FSI problems in $2.5 \mathrm{D}$ theory. And after the stress response comparison analysis, conclusions are shown as follows:The 2.5D FSI models had good enough simulation for 3D model on downstream sideline stress of dam bottom to replace the 3D model to do large-scale 3D calculation;2.5D model with 12-shells had better simulation of 3D model than models with 6 and 24 shells on downstream sideline stress of dam bottom.

\section{References}

[1] Bilodeau, William L, Bilodeau Sally W, Gath Eldon M (2007) Geology of Los Angeles, California ,United States of America, ENVIRONMENTAL \& ENGINEERING GEOSCIENCE,13(2) 99-160;

[2] Jordan Thomas H, Chen Yun-Tai, Gasparini Paolo (2011) Operational Earthquake Forecasting: State of Knowledge and Guidelines for Utilization, ANNALS OF GEOPHYSICS, 54(4), 315391 ;

[3] Graizer Vladimir (2006) Tilts in strong ground motion, BULLETIN OF THE SEISMOLOGICAL SOCIETY OF AMERICA, 96(6), 2090-2102;

[4] Zhou Qing, Zhang Chunshan, Chen Xianchenq (2011) Asymmetric disaster distribution and its cause analysis of the Ms8.0 Whenchuan earthquake, Acta Seismologica Sinic, 33(4), 492-504;

[5] Westergarrd (1993) Water Pressures on Dams During Earthquakes, Trans. Amer. Soc. Civ. 1933, 98:418-433;

[6] Liang Xu, Wang Zhenyu, Wang Lizhong (2015) A semi-analytical method to evaluate the dynamic response of functionally graded plates subjected to underwater shock, Journal of Sound and Vibration, v 336, 257-274;

[7] Javier Aviles, Xiangyue Li (1998) Analytical-numerical solution for hydrodynamic pressures on dams with sloping face considering compressibility and viscosity of water, Computer \& Structure, v56(4), 1998:481-488;

[8] Zienkiewjcz O.C., The finite element method, McGRAW-HILl, 1977;

[9] Ghaedi K, Jameel M , Ibrahim Z,et al. (2016) Seismic analysis of Roller Compacted Concrete (RCC) dams considering effect of sizes and shapes of galleries. KSCE JOURNAL OF CIVIL ENGINEERING, 20(1), 261-272;

[10] Puigdellivol O, Meresse D, Le Menach Y (2017) Thermal Topology Optimization of a ThreeLayer Laminated Busbar for Power Converters, IEEE TRANSACTIONS ON POWER ELECTRONICS, 32(6), 4691-4699 